\title{
Covers $\mathcal{P}$ for Abstract Regular Polytopes $\mathcal{Q}$ such that $\mathcal{Q}=\mathcal{P} / \mathbf{Z}_{p}^{k}$
}

\author{
Michael I. Hartley
}

Received: 9 June 2009 / Revised: 23 November 2009 / Accepted: 23 November 2009 /

Published online: 3 December 2009

(C) Springer Science+Business Media, LLC 2009

\begin{abstract}
One key problem in the theory of abstract polytopes is the so-called amalgamation problem. In its most general form, this is the problem of characterising the polytopes with given facets $\mathcal{K}$ and vertex figures $\mathcal{L}$. The first step in solving it for particular $\mathcal{K}$ and $\mathcal{L}$ is to find the universal such polytope, which covers all the others. This article explains a construction that may be attempted on an arbitrary polytope $\mathcal{P}$, which often yields an infinite family of finite polytopes covering $\mathcal{P}$ and sharing its facets and vertex figures. The existence of such an infinite family proves that the universal polytope is infinite; alternatively, the construction can produce an explicit example of an infinite polytope of the desired type. An algorithm for attempting the construction is explained, along with sufficient conditions for it to work. The construction is applied to a few $\mathcal{K}$ and $\mathcal{L}$ for which it was previously not known whether or not the universal polytope was infinite, or for which only a finite number of finite polytopes was previously known. It is conjectured that the construction is quite broadly applicable.
\end{abstract}

Keywords Abstract regular polytope - Covers of polytopes · Elementary Abelian group

\section{Introduction}

The study of regular polytopes has a long history, dating back to the dawn of mathematics. Progress in the field has normally come when researchers adopt a broader understanding of the objects, for example Kepler's understanding that stellations were as worthy of study as the platonic solids, or Schläfli's discovery of the four- and higher-dimensional regular convex polytopes. Over the last 30 years, the field has

M.I. Hartley (凶)

DownUnder Geosolutions, 80 Churchill Ave., Subiaco, 6008, Australia

e-mail: mikeh@dugeo.com 
been revived yet again through the study of what are called abstract regular polytopes, beginning with beautiful discoveries such as Coxeter and Grünbaum's 11-cell ([3] and [4]).

An abstract regular polytope is a purely combinatorial object whose definition is inspired by properties of the face-lattices of regular convex polytopes and tesselations of manifolds. Specifically, an abstract polytope $\mathcal{P}$ is a poset with unique minimal and maximal elements, and for which there exists a rank function mapping elements of the poset onto $\{-1, \ldots, n\}$. This rank function is bijective from any totally ordered subset of the poset to $\{-1, \ldots, n\}$. The rank of the poset will be the rank of its maximal element, that is, $n$. In the polytopes literature, a maximal totally ordered subset is called a flag.

Intervals (that is, subsets of the form $F / G=\{H: G \leq H \leq F\}$, where $G<F$ ) may be defined. These are called sections of the abstract polytope, and will themselves have an associated rank function. A second condition that an abstract polytope must satisfy is that if $\operatorname{rank} F=\operatorname{rank} G+2$, then the section $F / G$ contains only two elements besides $F$ and $G$ themselves.

Third and finally, abstract polytopes are required to be strongly connected. That is, for any two flags $\Phi, \Phi^{\prime}$ of a polytope, there must be a sequence of flags $\Phi=$ $\Phi_{0}, \ldots, \Phi_{m}=\Phi^{\prime}$ where for any $i$, the flags $\Phi_{i}$ and $\Phi_{i+1}$ differ by exactly one element, and each $\Phi_{i}$ contains $\Phi \cap \Phi^{\prime}$.

The sections of an abstract polytope are themselves abstract polytopes. Let $P_{-1}$ and $P_{n}$ be the unique elements of rank -1 and $n$ respectively. If $F$ and $G$ have ranks $n-1$ and 0 respectively, the sections $F / P_{-1}$ and $P_{n} / G$ are called (respectively) a facet and a vertex figure of $\mathcal{P}$.

A regular abstract polytope $\mathcal{P}$ is one whose automorphism group $\operatorname{Aut}(\mathcal{P})$ acts transitively on the set of its flags. Since this article is concerned exclusively with regular abstract polytopes, the term polytope will mean here a regular abstract polytope unless otherwise noted.

Given a base flag $\Phi$ of a polytope $\mathcal{P}$, the automorphism group $\operatorname{Aut}(\mathcal{P})$ is generated by $\left\langle\sigma_{0}, \ldots, \sigma_{n-1}\right\rangle$, where $\sigma_{i}$ is the element of $\operatorname{Aut}(\mathcal{P})$ that maps $\Phi$ to a flag differing from $\Phi$ by only an element of rank $i$. When written in this way, the automorphism group is a string $C$-group, that is, the $\sigma_{i}$ are involutions, $\sigma_{i}$ and $\sigma_{j}$ commute if $i \neq j \pm 1$, and the group satisfies the intersection property, that $\left\langle\sigma_{k}: k \in I\right\rangle \cap\left\langle\sigma_{k}: k \in J\right\rangle=\left\langle\sigma_{k}: k \in I \cap J\right\rangle$.

In fact, string $\mathrm{C}$-groups (with distinguished generators) and abstract regular polytopes are in one-to-one correspondence-a polytope $\mathcal{P}$ gives rise to a string $\mathrm{C}$-group $\Gamma(\mathcal{P})$ as its automorphism group, and a string C-group $\Gamma$ gives rise to a polytope $\mathcal{P}(\Gamma)$ as its coset geometry, the set of cosets of subgroups $\Gamma_{i}=\left\langle\sigma_{k}: k \neq i\right\rangle$ with incidence defined by nonemptiness of intersection. The fundamental theorem of abstract regular polytopes states that $\mathcal{P}(\Gamma(\mathcal{P})) \cong \mathcal{P}$ for any abstract regular polytope $\mathcal{P}$, and $\Gamma(\mathcal{P}(\Gamma)) \cong \Gamma$ for any string $\mathrm{C}$-group $\Gamma$. That is, the search for abstract polytopes is equivalent to the search for string $\mathrm{C}$-groups. Readers interested in more details are invited to study the field's canonical reference [11].

An important problem in the field is the so-called amalgamation problem. This may be phrased as a series of questions about particular polytopes $\mathcal{K}$ and $\mathcal{L}$, such as 
- Do there exist polytopes with facets $\mathcal{K}$ and vertex figures $\mathcal{L}$ ?

- If so, are all such polytopes finite?

- What finite examples are there?

The class of all polytopes with facets $\mathcal{K}$ and vertex figures $\mathcal{L}$ is denoted $\langle\mathcal{K}, \mathcal{L}\rangle$. All polytopes in $\langle\mathcal{K}, \mathcal{L}\rangle$ are quotients of a universal such polytope, denoted $\{\mathcal{K}, \mathcal{L}\}$. The three questions above can then be rephrased: Does $\{\mathcal{K}, \mathcal{L}\}$ exist? Is it finite? What finite quotients does it have?

If the automorphism groups of $\mathcal{K}$ and $\mathcal{L}$ are expressed as finitely presented groups, the presentation of $\operatorname{Aut}(\{\mathcal{K}, \mathcal{L}\})$ is easily determined; see Proposition 4A8 of [11] for details. The difficulty is then to figure out whether or not this presentation yields a C-group at all, and if it does, whether the polytope derived from it does indeed have the correct facets and vertex figures.

There is no general approach yet known to answer the questions of the amalgamation problem. However, some progress has been made for specific classes of $\mathcal{K}$ and $\mathcal{L}$. In particular, the finite universal locally toroidal polytopes have been characterised, except for a few special cases. These are polytopes where $\mathcal{K}$ and $\mathcal{L}$ are tesselations of toroids or spheres, but not both of spheres. See Chap. 8 to 12 of [11] for full details.

One important general result is that if the automorphism group of $\{\mathcal{K}, \mathcal{L}\}$ is an infinite "residually finite" group (for example, a linear group), then there exist infinitely many finite polytopes in $\langle\mathcal{K}, \mathcal{L}\rangle$ (see Theorem $4 \mathrm{C} 4$ of [11]). This proves, for example, that there are infinitely many finite locally spherical polytopes of types $\{4,3,5\}$, $\{3,5,3\}$ and $\{5,3,5\}$. Unfortunately, the proof is not a constructive one, so that although there are infinitely many finite polytopes of types $\{3,5,3\}$ and $\{5,3,5\}$, only a finite number were explicitly known until very recently.

This article shows a method of constructing new polytopes from known ones. For a given polytope $\mathcal{P} \in\langle\mathcal{K}, \mathcal{L}\rangle$ with automorphism group $\Gamma$, the construction gives a polytope $\mathcal{R} \in\langle\mathcal{K}, \mathcal{L}\rangle$ with automorphism group $\Gamma \ltimes V$, where $V$ is a direct product of cyclic groups. In some cases, there is a lot of flexibility in the choice of $V$. The method is applied to give explicit constructions of infinite families of polytopes of types $\{3,5,3\}$ and $\{5,3,5\}$, and to give the first proofs that certain locally toroidal universal polytopes are infinite. These examples are given to show that the technique, while simple, is actually quite powerful.

The structure of this article is as follows. Section 2 outlines the construction and gives sufficient conditions for it to work. Section 3 applies the construction to a number of special cases. Section 4 considers algorithmic questions useful to those wishing to apply the construction in a systematic way.

\section{Defining the Construction}

Let $\mathcal{P}$ be an abstract regular polytope, with automorphism group $\Gamma$ generated by $\left\{\sigma_{0}, \ldots, \sigma_{n-1}\right\}$. Let $R$ and $S$ be sets of words on $\left\{\sigma_{0}, \ldots, \sigma_{n-2}\right\}$ and $\left\{\sigma_{1}, \ldots, \sigma_{n-1}\right\}$ respectively, complete sets of defining relations (including standard relations) for the automorphism groups $\left\langle\sigma_{0}, \ldots, \sigma_{n-2}\right\rangle$ and $\left\langle\sigma_{1}, \ldots, \sigma_{n-1}\right\rangle$ of the facets and vertex figures of $\mathcal{P}$. 
Suppose $\Gamma$ acts on the set $\{1, \ldots, k\}$, and let $U$ be the direct product of $k$ cyclic groups of the same order, say $U=\left\langle e_{1}, \ldots, e_{k}\right\rangle$. We can define a multiplication between elements of $\Gamma$ and elements of $U$ via $e_{i} \sigma_{j}=\sigma_{j} e_{k}$, where $k$ is the image of $i$ under the action of $\sigma_{j}$. This defines a semidirect product $\Gamma \ltimes U$ of $\Gamma$ with $U$.

Let $u_{0}, \ldots, u_{n-1} \in U$, and let $s_{i}=\sigma_{i} u_{i}$ for all $i$. In general, the $s_{i}$ will not be involutions. If they are, they will not in general generate a $\mathrm{C}$-group. However, the next theorem gives sufficient conditions for $\left\langle s_{0}, \ldots, s_{n-1}\right\rangle$ to be a string C-group, the group of a regular abstract polytope.

Theorem 2.1 Let $\Gamma, \sigma_{i}, U, s_{i}, R$ and $S$ be defined as above. Suppose further that $\left(s_{0} s_{n-1}\right)^{2}=1$ if $n>2$ and that $s_{i_{1}} s_{i_{2}} \cdots s_{i_{t}}=1$ for any word $\sigma_{i_{1}} \sigma_{i_{2}} \cdots \sigma_{i_{t}}$ in $R \cup S$. Then $W=\left\langle s_{0}, \ldots, s_{n-1}\right\rangle$ is a string $C$-group.

Proof The $s_{i}$ are all involutions, since $R \cup S$ contains the relations (words) $\sigma_{i}^{2}$ for all $i$. Likewise, $\left(s_{i} s_{j}\right)^{2}=1$ whenever $i \neq j \pm 1$, since the case $\{i, j\}=\{0, n-1\}$ is given explicitly in the statement of the theorem, and the other cases are derived from the fact that $\left(\sigma_{i} \sigma_{j}\right)^{2}=1$ whenever $i \neq j \pm 1$. It follows that $W$ is a group generated by involutions, with a string diagram. It remains to show that it satisfies the intersection property, that is, that $\left\langle s_{k}: k \in I\right\rangle \cap\left\langle s_{k}: k \in J\right\rangle=\left\langle s_{k}: k \in I \cap J\right\rangle$. It is, in fact, sufficient to prove this for the case where $I$ and $J$ are intervals - sets of the form $\left\{k: k_{0} \leq k \leq k_{1}\right\}$.

Let $x \in W$ be such that $x \in\left\langle s_{k}: k \in I\right\rangle \cap\left\langle s_{k}: k \in J\right\rangle$ but $x \notin\left\langle s_{k}: k \in I \cap J\right\rangle$, where $I$ and $J$ are intervals. Without loss of generality, assume that neither $I$ nor $J$ is $\{0, \ldots, n-1\}$. If $x=s_{k_{1}} s_{k_{2}} \cdots s_{k_{a}}=s_{k_{1}^{\prime}} s_{k_{2}^{\prime}} \cdots s_{k_{b}^{\prime}}$, where the $k_{i} \in I$ and the $k_{i}^{\prime} \in J$, then $x=\sigma_{k_{1}} \sigma_{k_{2}} \cdots \sigma_{k_{a}} v=\sigma_{k_{1}^{\prime}} \sigma_{k_{2}^{\prime}} \cdots \sigma_{k_{b}^{\prime}} v^{\prime}$ for some $v, v^{\prime} \in U$. It follows that $\sigma_{k_{b}} \cdots \sigma_{k_{1}^{\prime}} \sigma_{k_{1}} \cdots \sigma_{k_{a}}=v^{-1} v^{\prime} \in U \cap \Gamma=\{1\}$. This shows that $v=v^{\prime}$ and that $\sigma_{k_{1}} \sigma_{k_{2}} \cdots \sigma_{k_{a}}=\sigma_{k_{1}^{\prime}} \sigma_{k_{2}^{\prime}} \cdots \sigma_{k_{b}^{\prime}}$. These equalities imply that $x$ may be written $\sigma_{l_{1}} \cdots \sigma_{l_{c}} v$, where the $l_{i}$ are all elements of $I \cap J$. If $I$ is an interval not equal to $\{0, \ldots, n-1\}$, any word on $\left\{\sigma_{i}: i \in I\right\}$ that equals 1 in $\Gamma$ may be reduced to the empty word using only relations in either $R$ or $S$. In particular, this holds for $\sigma_{l_{1}} \cdots \sigma_{l_{c}}\left(\sigma_{k_{1}} \cdots \sigma_{k_{a}}\right)^{-1}$, and therefore $s_{l_{1}} \cdots s_{l_{c}}\left(s_{k_{1}} \cdots s_{k_{a}}\right)^{-1}$ equals 1 in $W$. It follows that $x \in\left\langle s_{k}: k \in I \cap J\right\rangle$, as required.

$W=\left\langle s_{0}, \ldots, s_{n-1}\right\rangle$ will not usually equal $\Gamma \ltimes U$, but will instead equal $\Gamma \ltimes V$ for some subgroup $V$ of $U$. In fact, $V$ might be the trivial subgroup, which would not be terribly interesting. However, there is no particular reason why it should always be trivial. The following is worth noting.

Theorem 2.2 If $W=\Gamma \ltimes V$ is a string $C$-group, the polytope $\mathcal{P}(W)$ covers $\mathcal{P}=$ $\mathcal{P}(\Gamma)$. In fact, $\mathcal{P} \cong \mathcal{P}(W) / V$.

Proof It is clear from the definitions that $V$ is normal in $W$, and the quotient $W / V \cong \Gamma$. The result then follows from Proposition $2 \mathrm{E} 18$ of [11].

Theorem 2.3 Let $\Gamma, \sigma_{i}, U, s_{i}, R$ and $S$ satisfy the conditions of Theorem 2.1. Then $\mathcal{P}(W)$ has facets and vertex figures isomorphic to those of $\mathcal{P}(\Gamma)$. 
Proof The facets of $\mathcal{P}(W)$ and $\mathcal{P}(\Gamma)$ have groups generated by $\left\langle s_{0}, \ldots, s_{n-2}\right\rangle$ and $\left\langle\sigma_{0}, \ldots, \sigma_{n-2}\right\rangle$ respectively. These groups are isomorphic, since $\left\{s_{0}, \ldots, s_{n-2}\right\}$ satisfies all the same relations as $\left\langle\sigma_{0}, \ldots, \sigma_{n-2}\right\rangle$, and so the facets are isomorphic. Similarly, the vertex figures are isomorphic.

Before further developing the general theory of this construction, it is worth applying it to certain previously unresolved problems in the theory of abstract polytopes.

\section{Applications of the Construction}

It is known that there are infinitely many finite polytopes of type $\{5,3,5\}$ and $\{3,5,3\}$. However, until the appearance of [12], only a handful of examples appeared in the literature. Coxeter and Grünbaum independently discovered the 11-cell, a self-dual locally projective polytope of type $\{3,5,3\}$, in [3] and [4]. Coxeter followed this with the discovery of the self-dual, locally projective 57-cell, of type $\{5,3,5\}$, in [2]. Work by Hartley and Leemans [7-9] gave some sporadic examples of polytopes of type $\{5,3,5\}$ related to the 57 -cell and its locally projective covers. Some other locally spherical polytopes of these types, including the polytopes $\mathcal{P}_{1}$ and $\mathcal{P}_{2}$ defined below, appeared in [5]. The polytopes $\mathcal{P}_{1}$ and $\mathcal{P}_{3}$ (again, see below) appear in [10]. In [12] numerous infinite families of polytopes are constructed by finding a representation of the group of an infinite universal polytope as a linear group over a ring and then taking finite quotients of the ring. In particular, infinite families of locally spherical polytopes of types $\{3,5,3\}$ and $\{5,3,5\}$ are given. The infinite families constructed in the next few paragraphs do not coincide with those in [12] (in fact, there is almost no overlap), as may be noted by comparing the orders of the respective automorphism groups.

Let $\mathcal{P}_{1}$ be the regular polytope with automorphism group $\Gamma_{1}=\left\langle\sigma_{0}, \sigma_{1}, \sigma_{2}, \sigma_{3}\right\rangle$, where

- $\sigma_{0}=(4,6)(7,10)(9,12)(11,15)(13,14)(16,17)$,

- $\sigma_{1}=(2,4)(5,7)(6,9)(8,11)(10,14)(15,17)$,

- $\sigma_{2}=(1,2)(3,5)(9,13)(11,16)(12,14)(15,17)$, and

- $\sigma_{3}=(1,3)(5,8)(7,11)(10,15)(13,16)(14,17)$.

It may be verified easily enough that $\mathcal{P}_{1}$ is a regular abstract polytope with dodecahedral facets and icosahedral vertex figures. Its automorphism group has order 8160 and a normal subgroup of index 2, isomorphic to the projective special linear group $L_{2}(16)$. This particular representation of $\Gamma_{1}$ was found, using [13], by taking the quotient of $[5,3,5]$ by the core of an index 17 subgroup.

Let $U_{1}$ be the direct product of 17 cyclic groups of order $p$, say $U_{1}=\left\langle e_{1}, \ldots, e_{17}\right\rangle$, and let $s_{0}=\sigma_{0}, s_{1}=\sigma_{1} e_{2} e_{4}^{-1}, s_{2}=\sigma_{2}$ and $s_{3}=\sigma_{3}$, where the multiplication between the $\sigma_{i}$ and the $e_{j}$ is as outlined in the previous section.

Theorem 3.1 Suppose 3 does not divide $p$. Then the group $\left\langle s_{0}, s_{1}, s_{2}, s_{3}\right\rangle$ is a string $C$-group equal to $\Gamma_{1} \ltimes \mathbf{Z}_{p}^{16}$, the group of a regular abstract polytope of type $\{5,3,5\}$. 
Proof It may be verified that these $s_{i}$ satisfy the conditions of Theorem 2.1 (mostly, trivially), and therefore (by Theorems 2.1 and 2.3) $\left\langle s_{0}, s_{1}, s_{2}, s_{3}\right\rangle$ is a string C-group, the group of a polytope of type $\{5,3,5\}$. Note next that

$$
\begin{aligned}
s_{3} s_{2} & =(1,5,8,3,2)(7,16,9,13,11)(10,17,12,14,15), \\
s_{3} s_{2} s_{1} & =(1,7,16,6,9,13,8,3,4,2)(5,11)(10,15,14,17,12) e_{2} e_{4}^{-1}, \quad \text { and } \\
s_{3} s_{2} s_{1} s_{0} & =(1,10,11,5,15,13,8,3,6,12,7,17,9,14,16,4,2) e_{2} e_{6}^{-1} .
\end{aligned}
$$

From this it follows that

$$
\begin{aligned}
\left(s_{3} s_{2} s_{1} s_{0} s_{3} s_{2} s_{1}\right)= & (1,15,8,4)(2,7,12,16)(3,9,17,13) \\
& \times(5,14,6,10) e_{1} e_{2} e_{4}^{-1} e_{9}^{-1}, \text { so } \\
\left(s_{3} s_{2} s_{1} s_{0} s_{3} s_{2} s_{1}\right)^{2}= & (1,8)(15,4)(2,12)(7,16)(3,17)(9,13)(5,6) \\
& \times(14,10) e_{2} e_{4}^{-1} e_{7} e_{9}^{-1} e_{15} e_{17}^{-1}, \quad \text { and } \\
\left(s_{3} s_{2} s_{1} s_{0} s_{3} s_{2} s_{1}\right)^{2} s_{3} s_{2}= & (1,3,12)(2,14,17)(4,10,15)(5,6,8) \\
& \times(7,9,11) e_{1} e_{4}^{-1} e_{10} e_{12}^{-1} e_{13}^{-1} e_{16} .
\end{aligned}
$$

Cubing this gives

$$
\begin{aligned}
\left(\left(s_{3} s_{2} s_{1} s_{0} s_{3} s_{2} s_{1}\right)^{2} s_{3} s_{2}\right)^{3}= & \left(e_{12} e_{15}^{-1} e_{4} e_{3}^{-1} e_{13}^{-1} e_{16}\right)\left(e_{3} e_{10}^{-1} e_{15} e_{1}^{-1} e_{13}^{-1} e_{16}\right) \\
& \times\left(e_{1} e_{4}^{-1} e_{10} e_{12}^{-1} e_{13}^{-1} e_{16}\right)
\end{aligned}
$$

which equals $e_{13}^{-3} e_{16}^{3}$.

Conjugating an element of $U_{1}$ by $s_{i}$ is equivalent to conjugating it by $\sigma_{i}$. Therefore, knowing that $\Gamma_{1}$ acts 2-transitively (in fact, 3-transitively) on $\{1, \ldots, 17\}$ and that 3 does not divide $p$, it follows that $W_{1}$ contains $e_{j} e_{k}^{-1}$ for all $j$ and $k$ (in particular for $j=2$ and $k=4)$, so that $W_{1}$ contains $\left\langle\sigma_{0}, \ldots, \sigma_{3}, e_{1} e_{2}^{-1}, e_{2} e_{3}^{-1}, \ldots, e_{16} e_{17}^{-1}\right\rangle$. That $W_{1}$ in fact equals the latter may be shown by noting that it contains all the generators of $W_{1}$. This completes the proof.

It follows that the groups $W_{1}=W_{1}^{(p)}$ form an infinite family of automorphism groups of finite polytopes $\mathcal{P}_{1}^{(p)}$ with Schläfli type $\{5,3,5\}$. In fact, even if 3 does divide $p$, the construction given above yields nontrivial covers of $\mathcal{P}_{1}$.

Another infinite family of the same type may be derived from the polytope $\mathcal{P}_{2}$ with automorphism group $\Gamma_{2}=\left\langle\sigma_{0}, \sigma_{1}, \sigma_{2}, \sigma_{3}\right\rangle$, given by

- $\sigma_{0}=(3,5)(6,9)(8,13)(10,15)(11,18)(14,20)(16,24)(17,23)(19,21)(22,28)$

$(25,30)(26,32)(27,33)(29,34)(31,35)$,

- $\sigma_{1}=(2,3)(4,6)(5,8)(7,11)(9,14)(10,16)(12,19)(15,22)(17,24)(18,26)$

$(21,27)(23,29)(25,31)(28,34)(35,36)$,

- $\sigma_{2}=(1,2)(4,7)(6,10)(8,13)(9,15)(11,16)(14,21)(17,25)(18,24)(19,20)$ $(22,27)(23,30)(26,31)(28,33)(32,35)$, and 
- $\sigma_{3}=(2,4)(3,6)(5,9)(7,12)(8,14)(10,17)(11,19)(13,20)(15,23)(16,24)$

$(18,21)(22,29)(26,27)(28,34)(32,33)$.

This is easily verified to be the group of a locally spherical polytope of type $\{5,3,5\}$. It has order 7200 and an index 2 normal subgroup isomorphic to $A_{5} \times A_{5}$. As for $\mathcal{P}_{1}$, this polytope is not new. Again as for $\mathcal{P}_{1}$, this particular representation of $\Gamma_{2}$ was found using [13], by taking the quotient of $[5,3,5]$ by the core of an index 36 subgroup.

Let $U_{2}$ be $\mathbf{Z}_{p}^{36}$, generated by $\left\{e_{1}, \ldots, e_{36}\right\}$, let $s_{1}=\sigma_{1} e_{5}^{-1} e_{8} e_{9}^{-1} e_{14} e_{15}^{-1} e_{22} e_{23}^{-1}$ $e_{29} e_{35} e_{36}^{-1}$, and let $s_{i}=\sigma_{i}$ for $i \neq 1$.

Theorem 3.2 Suppose $p$ is odd. Then the group $W_{2}=\left\langle s_{0}, s_{1}, s_{2}, s_{3}\right\rangle$ is a string $C$ group equal to $\Gamma_{2} \ltimes \mathbf{Z}_{p}^{25}$, the group of a regular abstract polytope of type $\{5,3,5\}$.

Proof Again, it is straightforward to verify that the conditions of Theorem 2.1 are satisfied. It is also straightforward to show that $\left(\left(s_{0} s_{1} s_{2} s_{3}\right)^{3} s_{2}\right)^{4}=\left(e_{6} e_{10} e_{12}^{-1} e_{34}^{-1}\right)^{4}$, and therefore (since $p$ is odd) that $e_{6} e_{10} e_{12}^{-1} e_{34}^{-1} \in W_{2}$, and so also is every conjugate of the latter by elements of $\Gamma$. It may be computationally verified that these 450 conjugates generate a 25-dimensional subset of $U_{2}$, which contains $e_{5}^{-1} e_{8} e_{9}^{-1} e_{14} e_{15}^{-1} e_{22} e_{23}^{-1} e_{29} e_{35} e_{36}^{-1}$. It follows that $W_{2}$ is as claimed.

In fact, even if $p$ is even, $W_{2}$ is a string C-group, and its polytope properly covers $\mathcal{P}_{2}$. However, it is smaller than $\Gamma_{2} \ltimes \mathbf{Z}_{p}^{25}$ would be.

The next example is of an infinite family of polytopes of type $\{3,5,3\}$. Let $\mathcal{P}_{3}$ be the regular polytope with automorphism group $\Gamma_{3}=\left\langle\sigma_{0}, \sigma_{1} \sigma_{2}, \sigma_{3}\right\rangle$, where

- $\sigma_{0}=(4,7)(8,12)(9,13)(11,16)(14,19)(15,20)(17,21)(18,23)(22,26)(24,25)$,

- $\sigma_{1}=(2,4)(5,9)(6,11)(8,14)(10,15)(12,18)(17,22)(19,23)(21,25)(24,26)$,

- $\sigma_{2}=(1,2)(3,6)(4,8)(5,10)(7,12)(11,17)(15,20)(16,21)(18,24)(23,25)$, and

- $\sigma_{3}=(1,3)(2,5)(4,9)(6,10)(7,13)(11,15)(16,20)(17,21)(22,25)(24,26)$.

It is easily verified that these generate a 2-transitive string C-group, the automorphism group of a locally spherical polytope of type $\{3,5,3\}$. The group has order 15600 and has the projective special linear group $L_{2}(25)$ as an index 2 normal subgroup. Let $U_{3}=\left\langle e_{1}, \ldots, e_{26}\right\rangle$ be the direct product of 26 copies of the cyclic group of order $p$. Finally, let $s_{2}=\sigma_{2} e_{1} e_{2}^{-1} e_{5}^{-1} e_{10}$, and let $s_{i}=\sigma_{i}$ for $i \neq 2$. This representation of $\Gamma_{3}$ was found in a similar way to $\Gamma_{1}$ and $\Gamma_{2}$.

Theorem 3.3 Suppose $p$ is odd. Then the group $W_{3}=\left\langle s_{0}, s_{1}, s_{2}, s_{3}\right\rangle$ is a string $C$ group equal to $\Gamma_{3} \ltimes \mathbf{Z}_{p}^{25}$, the group of a regular abstract polytope of type $\{3,5,3\}$.

Proof As before, it is straightforward to check the conditions of Theorem 2.1. Then, note that $\left(\left(s_{3} s_{2} s_{1}\right)^{4} s_{0} s_{2} s_{1}\right)^{4}=e_{8}^{4} e_{23}^{-4}$, so that (since $p$ is odd and $\Gamma_{3}$ acts 2-transitively on the $\left.e_{i}\right) e_{i} e_{j}^{-1} \in W_{3}$ for all $i, j$. In particular, $e_{1} e_{2}^{-1} e_{5}^{-1} e_{10} \in W_{3}$, from which it follows that $W_{3}=\left\langle\sigma_{0}, \ldots, \sigma_{3}, e_{1} e_{2}^{-1}, \ldots, e_{25} e_{26}^{-1}\right\rangle$. This completes the proof. 
The $\mathcal{P}\left(W_{3}\right)=\mathcal{P}\left(W_{3}^{(p)}\right)$ for $p$ odd thus form an infinite family of finite polytopes of type $\{3,5,3\}$. In fact, even if $p$ is even, $\mathcal{P}\left(W_{3}\right)$ is a polytope properly covering $\mathcal{P}_{3}$. However, it is no longer a $p^{25}$-fold cover.

The same construction may be applied to yield an infinite family of finite polytopes of type $\{4,3,5\}$. However, another such an infinite family is already provided by the long-known twisting operation described in Chap. 8 of [11]. For example, if $\mathcal{G}=$ $\mathcal{G}_{p}$ is the graph consisting of 6 edges labelled $p$, connecting antipodal points of an icosahedron, then $2^{\{3,5\}, \mathcal{G}}$ will be a finite polytope of type $\{4,3,5\}$, with a group isomorphic to $D_{2 p}^{6} \rtimes \Gamma(\{3,5\})$.

The above paragraphs demonstrate three infinite families of finite polytopes of two Schläfli types for which, until very recently, only a finite number of finite polytopes were known. In the next few paragraphs, three universal polytopes will be shown to be infinite. It was previously not known whether or these universal polytopes were finite or infinite.

Let $\mathcal{P}_{4}$ be the polytope with group generated by

- $\sigma_{0}=(1,2)(3,5)(4,7)(6,10)(8,12)(9,13)(11,16)(14,18)(15,20)(17,23)$

$(19,26)(21,27)(22,28)(24,30)(25,31)(29,33)(32,34)(35,37)(36,38)$,

- $\sigma_{1}=(2,4)(5,9)(10,15)(12,17)(14,19)(16,22)(18,25)(21,26)(27,31)(33,36)$,

- $\sigma_{2}=(1,2)(3,6)(4,8)(5,10)(7,12)(9,14)(11,16)(13,18)(15,21)(17,24)$

$(19,26)(20,27)(22,29)(23,30)(25,32)(28,33)(31,34)(35,36)(37,38)$, and

- $\sigma_{3}=(1,3)(2,5)(4,9)(6,11)(7,13)(10,16)(15,22)(20,28)(32,35)(34,37)$.

This $\mathcal{P}_{4}$ is a locally toroidal polytope with Schläfli type $\{4,4,4\}$. Its facets and vertex figures are tesselations of tori by squares, $15^{2}$ squares per facet, $9^{2}$ squares per vertex figure. More succinctly, using the notation of Section 10C of [11], $\mathcal{P}_{4}$ is a quotient of the universal ${ }_{2} \mathcal{T}_{(15,0),(9,0)}$ with the same facets and vertex figures. (The polytope ${ }_{2} \mathcal{T}_{(a, 0),(b, 0)}$ is defined as the universal polytope $\left\{\{4,4\}_{(a, 0)},\{4,4\}_{(b, 0)}\right\}$ whose facets and vertex figures are the regular toroids $\{4,4\}_{(a, 0)}$ and $\{4,4\}_{(b, 0)}$ respectively.) The four generators $\sigma_{0}, \sigma_{1}, \sigma_{2}$ and $\sigma_{3}$ of $\Gamma_{4}=\operatorname{Aut}\left(\mathcal{P}_{4}\right)$ together induce every possible permutation of $\{1, \ldots, 38\}$, that is, $\Gamma_{4} \cong S_{38}$.

Let $U=\mathbf{Z}^{38}$, generated by $\left\{e_{1}, \ldots, e_{38}\right\}$, and let $s_{0}=\sigma_{0} e_{15}^{-1} e_{20} e_{22} e_{28}^{-1}, s_{1}=$ $\sigma_{1} e_{10} e_{15}^{-1}, s_{2}=\sigma_{2} e_{15}^{-1} e_{21} e_{22} e_{29}^{-1}$ and $s_{3}=\sigma_{3} e_{15}^{-1} e_{20} e_{22} e_{28}^{-1}$.

Theorem 3.4 The universal polytope ${ }_{2} \mathcal{T}_{(15,0),(9,0)}$ is infinite.

Proof Let $W_{4}=\left\langle s_{0}, s_{1}, s_{2}, s_{3}\right\rangle$. It is tedious, but straightforward, to verify that the conditions of Theorem 2.1 are satisfied, that is, that $s_{0}^{2}, s_{1}^{2}, s_{2}^{2}, s_{3}^{2},\left(s_{0} s_{1}\right)^{4},\left(s_{0} s_{2}\right)^{2}$, $\left(s_{0} s_{3}\right)^{2},\left(s_{1} s_{2}\right)^{4},\left(s_{1} s_{3}\right)^{2},\left(s_{2} s_{3}\right)^{2},\left(s_{0} s_{1} s_{2} s_{1}\right)^{15}$ and $\left(s_{2} s_{3} s_{4} s_{3}\right)^{9}$ are all equal to the identity in $W_{4}$.

Then, note that $\left(s_{0}\left(s_{1} s_{2} s_{3} s_{2}\right)^{2} s_{3}\right)^{12}$ is equal to $e_{25}^{-12} e_{38}^{12}$. It follows that $W_{4}$ is infinite, the automorphism group of an infinite polytope of type $\left\{\{4,4\}_{(15,0)}\right.$, $\left.\{4,4\}_{(9,0)}\right\}$.

Note that the universal polytopes of type $\left\{\{4,4\}_{(a, b)},\{4,4\}_{(c, d)}\right\}$ have been classified, except for those where $b=d=0$, and $a$ and $c$ are distinct odd integers (see [11] 
for details). This theorem therefore addresses one of the exceptional cases, as does the next.

The same construction may be applied to show that ${ }_{2} \mathcal{T}_{(21,0),(15,0)}$ is infinite. Let $\mathcal{P}_{5}$ be the polytope with group generated by

- $\sigma_{0}=(1,2)(3,5)(4,7)(6,10)(8,12)(9,13)(11,16)(14,17)(15,19)(18,23)$ $(20,24)(21,25)(22,27)(26,29)(28,30)(31,33)(32,35)(34,37)(36,38)$,

- $\sigma_{1}=(2,4)(5,9)(10,15)(14,18)(16,21)(17,22)(20,23)(24,27)(29,32)(34,38)$,

- $\sigma_{2}=(1,2)(3,6)(4,8)(5,10)(7,12)(9,14)(11,16)(13,17)(15,20)(18,23)$ $(19,24)(21,26)(22,28)(25,29)(27,30)(31,34)(32,36)(33,37)(35,38)$, and - $\sigma_{3}=(1,3)(2,5)(4,9)(6,11)(7,13)(10,16)(15,21)(19,25)(28,31)(30,33)$.

Then, let $s_{0}=\sigma_{0} e_{15}^{-1} e_{19} e_{21} e_{25}^{-1}, s_{1}=\sigma_{1} e_{10} e_{15}^{-1}, s_{2}=\sigma_{2} e_{15}^{-1} e_{20} e_{21} e_{26}^{-1}$ and $s_{3}=$ $\sigma_{3} e_{15}^{-1} e_{19} e_{21} e_{25}^{-1}$, where the $e_{i}$ are the generators of $U=\mathbf{Z}^{38}$.

Theorem 3.5 The universal polytope ${ }_{2} \mathcal{T}_{(21,0),(15,0)}=\left\{\{4,4\}_{(21,0)},\{4,4\}_{(15,0)}\right\}$ is infinite.

Proof It is easily verified that $\left\langle\sigma_{0}, \sigma_{1}, \sigma_{2}, \sigma_{3}\right\rangle$ is a C-group, that $\left\langle\sigma_{0}, \sigma_{1}, \sigma_{2}\right\rangle$ is the group of $\{4,4\}_{(21,0)}$, and $\left\langle\sigma_{1}, \sigma_{2}, \sigma_{3}\right\rangle$ is the group of $\{4,4\}_{(15,0)}$. As before, the group $W_{5}=\left\langle s_{0}, s_{1}, s_{2}, s_{3}\right\rangle$ satisfies the conditions of Theorem 2.1. Then, note that the element $s_{3}\left(s_{2} s_{1} s_{2} s_{3}\right)^{2}\left(s_{0} s_{1} s_{2} s_{1}\right) s_{0}$ of $W$ equals $e_{33}^{-8} e_{36}^{8}$. It follows that $W$ is an infinite $\mathrm{C}$-group, the automorphism group of a polytope of the stated type.

The constructions given above of polytopes of type $\left\{\{4,4\}_{(s, 0)},\{4,4\}_{(t, 0)}\right\}$ can be modified easily to give infinite families of finite polytopes of the specified types. The representations given for $\Gamma_{4}$ and $\Gamma_{5}$ were found in a similar manner to those of $\Gamma_{1}$, $\Gamma_{2}$ and $\Gamma_{3}$.

Finally, we turn to locally toroidal polytopes of type $\{3,6,3\}$. Section $11 \mathrm{H}$ of [11] shows a method of constructing a locally toroidal polytope of type $\{3,6,3\}$ from one of type $\{3,3,6\}$. In particular, the universal polytope $\left\{\{3,3\},\{3,6\}_{(s, 0)}\right\}(s \geq 2, s$ not a multiple of 3 ) yields a polytope of type $\left\{\{3,6\}_{(s, 0)},\{6,3\}_{(s, 0)}\right\}$ with the same size automorphism group.

For $s>4$, this shows that $\left\{\{3,6\}_{(s, 0)},\{6,3\}_{(s, 0)}\right\}={ }_{7} \mathcal{T}_{(s, 0),(s, 0)}^{4}$ is infinite. If $s=2$, it is finite, but for $s=4$, it is undecided. The polytope $\left\{\{3,3\},\{3,6\}_{(4,0)}\right\}$ is finite, with automorphism group of order 15360. The construction given in [11] therefore gives an example of a finite polytope of type $\left\{\{3,6\}_{(4,0)},\{6,3\}_{(4,0)}\right\}$, but beyond this, no clue as to how large or small the universal polytope might be. The automorphism group $\Gamma_{6}$ of this example can be expressed as $\left\langle\sigma_{0}, \sigma_{1}, \sigma_{2}, \sigma_{3}\right\rangle$, where

- $\sigma_{0}=(1,33)(2,34)(3,35)(4,36)(5,37)(6,38)(7,39)(8,40)(9,41)(10,42)$

$(11,43)(12,44)(13,45)(14,46)(15,47)(16,48)(21,49)(22,50)(23,51)(25,52)$

$(26,53)(27,54)(29,55)(31,56)(57,65)(58,66)(59,67)(61,68)(69,70)(71,72)$

$(75,79)(76,80)(77,81)(82,83)(84,85)(88,92)(89,93)(90,94)(95,96)(97,98)$

$(101,103)(104,105)(106,107)(111,113)(114,115)(116,117)(121,122)$

(123, 124), 
- $\sigma_{1}=(1,13)(2,14)(3,15)(5,16)(7,17)(8,18)(9,19)(10,20)(21,22)(23,24)$ $(25,26)(27,28)(33,57)(34,58)(35,59)(36,60)(37,61)(38,62)(43,63)(44,64)$ $(45,65)(46,66)(47,67)(48,68)(49,69)(50,70)(52,71)(53,72)(55,73)(56,74)$ $(75,77)(76,78)(79,82)(81,83)(84,86)(88,90)(89,91)(92,95)(94,96)(97,99)$ $(103,108)(105,109)(107,110)(113,118)(115,119)(117,120)(121,126)$ $(123,127)$,

- $\sigma_{2}=(1,7)(2,8)(3,4)(5,11)(6,12)(9,10)(13,29)(14,21)(15,26)(16,27)$ $(17,30)(18,20)(19,24)(22,23)(25,31)(28,32)(33,39)(34,40)(35,36)(37,43)$ $(38,44)(41,42)(45,55)(46,49)(47,53)(48,54)(50,51)(52,56)(57,88)(58,89)$ $(59,101)(60,102)(61,77)(62,78)(63,64)(65,92)(66,93)(67,103)(68,81)$ $(69,104)(70,105)(71,106)(72,107)(73,99)(74,100)(75,76)(79,80)(82,84)$ $(83,85)(86,87)(90,111)(91,112)(94,113)(95,121)(96,122)(97,123)(98,124)$ $(108,119)(109,125)(110,120)(114,116)(115,117)(118,128)(126,127)$, and

- $\sigma_{3}=(1,2)(3,5)(4,6)(7,9)(8,10)(11,12)(13,14)(15,16)(17,19)(18,20)$ $(21,25)(22,26)(23,27)(24,28)(29,31)(30,32)(33,34)(35,37)(36,38)(39,41)$ $(40,42)(43,44)(45,46)(47,48)(49,52)(50,53)(51,54)(55,56)(57,58)(59,61)$ $(60,62)(63,64)(65,66)(67,68)(69,71)(70,72)(73,74)(75,88)(76,89)(77,90)$ $(78,91)(79,92)(80,93)(81,94)(82,95)(83,96)(84,97)(85,98)(86,99)$ $(87,100)(101,111)(102,112)(103,113)(104,114)(105,115)(106,116)$ $(107,117)(108,118)(109,119)(110,120)(121,123)(122,124)(125,128)$ (126, 127).

This permutation representation for $\Gamma_{6}$ was found using GAP [13]. It is the action of $\Gamma_{6}$ on the cosets of one of its index 128 subgroups. The following theorem, conjectured in $11 \mathrm{H} 7$ of [11], may now be proven.

Theorem 3.6 The universal polytope ${ }_{7} \mathcal{T}_{(4,0),(4,0)}^{4}$ is infinite.

Proof Let $U=\mathbf{Z}^{128}$, generated by $\left\{e_{1}, \ldots, e_{128}\right\}$, let

$$
s_{1}=\sigma_{1}\left(e_{7} e_{9} e_{21} e_{25} e_{49} e_{52}\right)^{-1}\left(e_{17} e_{19} e_{22} e_{26} e_{69} e_{71}\right)
$$

and $s_{i}=\sigma_{i}$ for $i \neq 1$. Finally, let $W=\left\langle s_{0}, s_{1}, s_{2}, s_{3}\right\rangle$. It may be confirmed that $W$ satisfies the conditions of Theorem 2.1 and is therefore the group of a polytope of type $\left\{\{3,6\}_{(4,0)},\{6,3\}_{(4,0)}\right\}$. However, $W$ is infinite, as may be confirmed by noting that $\left(s_{0} s_{1} s_{2} s_{3} s_{0} s_{1} s_{2}\right)^{6}$ is a nonidentity element of $\left\langle e_{1}, \ldots, e_{128}\right\rangle$, and thus has infinite order.

To summarise, this section gives explicit constructions for new infinite families of finite polytopes of types $\{3,5,3\}$ and $\{5,3,5\}$, and proofs that three universal locally toroidal polytopes (whose sizes were previously unknown) are infinite.

The next section gives some general results about the construction, and tips on how to apply it effectively to obtain similar results. 


\section{General Results}

The construction depends sensitively on the particular permutation representation chosen for $\Gamma$. Different actions of $\Gamma$ on generating sets of different $\mathbf{Z}_{p}^{k}$ can lead to different families of covering polytopes. The construction might even work for one choice and fail for another. The next few results aim to remove this dependence of the construction on the choice of action.

If $\Gamma$ acts on the generators of $\mathbf{Z}_{p}^{k}$, it either acts transitively or nontransitively. If it acts nontransitively, the generators of $\mathbf{Z}_{p}^{k}$ may be partitioned into blocks, with $\Gamma$ acting transitively on each block. Each block generates a subgroup $\mathbf{Z}_{p}^{k_{j}}$ of $\mathbf{Z}_{p}^{k}$, and $\mathbf{Z}_{p}^{k}$ will be the direct product of all these subgroups. We have the following.

Theorem 4.1 If a set of $u_{i} \in \mathbf{Z}_{p}^{k}$ is found such that $s_{i}=\sigma_{i} u_{i}$ satisfy the conditions of Theorem 2.1, each set of projections $\left\{u_{i} \pi_{j}: i=0, \ldots, n-1\right\}$ (where $\pi_{j}$ is the projection homomorphism from $\mathbf{Z}_{p}^{k}$ to $\left.\mathbf{Z}_{p}^{k_{j}}\right)$ will be such that $s_{i}^{(j)}=\sigma_{i}\left(u_{i} \pi_{j}\right)$ also satisfies the conditions of Theorem 2.1.

Proof It is not hard to show that if $s_{i_{1}} \cdots s_{i_{k}}=u \in U=\mathbf{Z}_{p}^{k}$, then $s_{i_{1}}^{(j)} \cdots s_{i_{k}}^{(j)}=u \pi_{j}$. The result then follows immediately.

We can thus restrict our attention to transitive actions. This being so, the following well-known result from the theory of group actions is worth stating.

Theorem 4.2 Any transitive action of a group is isomorphic to the natural action of the group on the cosets of one of its subgroups.

Proof See any good book chapter on group actions, for example, Proposition 5.9 of [1].

This characterisation of the transitive actions of a group provides a systematic way to attempt the construction. We need only search through the conjugacy classes of subgroups of $\Gamma$, construct the natural action on its cosets, and attempt the construction, preferably (for computational reasons) beginning with subgroups of low index. In fact, we can say more than this. Instead of examining every subgroup, only one needs to be checked, the trivial subgroup.

Theorem 4.3 Let $\Gamma, \sigma_{i}, U, s_{i}, R$ and $S$ be as in Theorem 2.1. Then the polytope with automorphism group $\left\langle s_{0}, \ldots, s_{n-1}\right\rangle$ is isomorphic to one derived from the action of $\Gamma$ on its own elements by multiplication.

Proof The action of $\Gamma$ on the generators of $U$ is, by Theorem 4.2, isomorphic to an action of $\Gamma$ on the cosets of a subgroup $H$ of $\Gamma$. This associates a coset of $H$ with each generator of $U$. Denote the generator associated with $H g$ by $e_{H g}$, then, define a homomorphism $\phi$ from $\Gamma \ltimes U$ to $\Gamma \ltimes \mathbf{Z}_{p}^{|\Gamma|}=\left\langle\sigma_{i}, e_{x}^{\prime}: i=0, \ldots, n-1 ; x \in \Gamma\right\rangle$ via 
$\sigma_{i} \phi=\sigma_{i}$, and $e_{H g} \phi=\prod_{x \in H g} e_{x}^{\prime}$. This homomorphism is one-to-one and therefore provides the desired isomorphism from $\left\langle s_{0}, \ldots, s_{n-1}\right\rangle$ to its image.

It is therefore sufficient (although computationally infeasible for large $\Gamma$ ) to restrict attention to this action of $\Gamma$ on its own elements.

Once an action of $\Gamma$ has been identified, it is desirable to find elements $u_{i}$ of $U$ that ensure the $s_{i}$ generate a string C-group. The conditions of Theorem 2.1 provide sufficient conditions for this to occur. If the $u_{i}$ are written as

$$
u_{i}=\prod_{j} e_{j}^{\alpha_{i, j}}
$$

for unknown coefficients $\alpha_{i, j}$, each relator of $\left\{\sigma_{0}, \ldots, \sigma_{n-2}\right\}$ and of $\left\{\sigma_{1}, \ldots, \sigma_{n-1}\right\}$, as well as $\left(\sigma_{0} \sigma_{n-1}\right)^{2}$ for $n>2$, yields a linear equality over $\mathbf{Z}$ or $\mathbf{Z}_{p}$ that the $\alpha_{i, j}$ must satisfy. The other relators of $\left\{\sigma_{0}, \ldots, \sigma_{n-1}\right\}$ yield inequalities; in order for the construction to give interesting results, it is desirable to find $\alpha_{i, j}$ such that all the equalities are satisfied, as well as at least one of the inequalities. It is worth noting the following.

Theorem 4.4 Let $E$ be a set of homogeneous linear equalities with integer coefficients over variables $\alpha_{i, j}$, and let $\varepsilon$ be a homogeneous linear inequality over the same variables. If there exist values $\alpha_{i, j} \in \mathbf{Q}$ that satisfy $E \cup\{\varepsilon\}$, then there also exist values $\alpha_{i, j} \in \mathbf{Z}$ that satisfy them. Hence also, there are infinitely many $p$ for which there exist $\alpha_{i, j} \in \mathbf{Z}_{p}$ that satisfy the corresponding equations over $\mathbf{Z}_{p}$.

Proof Since the equations are homogeneous, the solution over $\mathbf{Q}$ can be converted to a solution over $\mathbf{Z}$ by multiplying by the lowest common multiple of the denominators. Projecting the latter solution into $\mathbf{Z}_{p}$ will still satisfy all the equalities and will satisfy the inequality for infinitely many $p$ also.

It is worthwhile to see an example. We shall attempt to find a family of covers for the digon. The digon has group $\mathbf{Z}_{2}^{2}$, of order 4 . Its automorphism group is $\left\langle\sigma_{0}, \sigma_{1}\right\rangle=\langle(1,2)(3,4),(1,3)(2,4)\rangle$. This representation of the group of the digon was chosen so that its action on $\{1,2,3,4\}$ is isomorphic to the transitive action of the group on its elements. Let $e_{i}, i \in\{1,2,3,4\}$, be the generators for $\mathbf{Z}^{4}$, and let $s_{0}=\sigma_{0}\left(e_{1}^{\alpha_{01}} e_{2}^{\alpha_{02}} e_{3}^{\alpha_{03}} e_{4}^{\alpha_{04}}\right)$ and $s_{1}=\sigma_{1}\left(e_{1}^{\alpha_{11}} e_{2}^{\alpha_{12}} e_{3}^{\alpha_{13}} e_{4}^{\alpha_{14}}\right)$.

The relators of the digon are simple enough. To satisfy the conditions of Theorem 2.1, we need $s_{0}^{2}=1$ and $s_{1}^{2}=1$. The first of these implies

$$
\begin{aligned}
& \left(\sigma_{0}\left(e_{1}^{\alpha_{01}} e_{2}^{\alpha_{02}} e_{3}^{\alpha_{03}} e_{4}^{\alpha_{04}}\right)\right)^{2}=1, \quad \text { that is, } \\
& \sigma_{0}^{2}\left(e_{2}^{\alpha_{01}} e_{1}^{\alpha_{02}} e_{4}^{\alpha_{03}} e_{3}^{\alpha_{04}} e_{1}^{\alpha_{01}} e_{2}^{\alpha_{02}} e_{3}^{\alpha_{03}} e_{4}^{\alpha_{04}}\right)=1,
\end{aligned}
$$

which is satisfied if and only if $\alpha_{01}+\alpha_{02}=0$ and $\alpha_{03}+\alpha_{04}=0$.

Considering $s_{1}^{2}=1$ gives

$$
\begin{aligned}
& \left(\sigma_{1}\left(e_{1}^{\alpha_{11}} e_{2}^{\alpha_{12}} e_{3}^{\alpha_{13}} e_{4}^{\alpha_{14}}\right)\right)^{2}=1, \quad \text { that is, } \\
& \sigma_{1}^{2}\left(e_{3}^{\alpha_{11}} e_{4}^{\alpha_{12}} e_{1}^{\alpha_{13}} e_{2}^{\alpha_{14}} e_{1}^{\alpha_{11}} e_{2}^{\alpha_{12}} e_{3}^{\alpha_{13}} e_{4}^{\alpha_{14}}\right)=1,
\end{aligned}
$$


which gives the equations $\alpha_{11}+\alpha_{13}=0$ and $\alpha_{12}+\alpha_{14}=0$.

As long as these equations are satisfied, $\left\langle s_{0}, s_{1}\right\rangle$ will be a string C-group, the automorphism group of a polygon. Next, note that

$$
\begin{aligned}
s_{0} s_{1} & =\sigma_{0}\left(e_{1}^{\alpha_{01}} e_{2}^{\alpha_{02}} e_{3}^{\alpha_{03}} e_{4}^{\alpha_{04}}\right) \sigma_{1}\left(e_{1}^{\alpha_{11}} e_{2}^{\alpha_{12}} e_{3}^{\alpha_{13}} e_{4}^{\alpha_{14}}\right) \\
& =\sigma_{0} \sigma_{1}\left(e_{3}^{\alpha_{01}} e_{4}^{\alpha_{02}} e_{1}^{\alpha_{03}} e_{2}^{\alpha_{04}}\right)\left(e_{1}^{\alpha_{11}} e_{2}^{\alpha_{12}} e_{3}^{\alpha_{13}} e_{4}^{\alpha_{14}}\right) \\
& =(1,4)(2,3)\left(e_{1}^{\alpha_{03}+\alpha_{11}} e_{2}^{\alpha_{04}+\alpha_{12}} e_{3}^{\alpha_{01}+\alpha_{13}} e_{4}^{\alpha_{02}+\alpha_{14}}\right) .
\end{aligned}
$$

Squaring this gives

$$
\begin{aligned}
& \left(e_{1}^{\alpha_{03}+\alpha_{11}} e_{2}^{\alpha_{04}+\alpha_{12}} e_{3}^{\alpha_{01}+\alpha_{13}} e_{4}^{\alpha_{02}+\alpha_{14}}\right)\left(e_{4}^{\alpha_{03}+\alpha_{11}} e_{3}^{\alpha_{04}+\alpha_{12}} e_{2}^{\alpha_{01}+\alpha_{13}} e_{1}^{\alpha_{02}+\alpha_{14}}\right), \quad \text { that is, } \\
& \left(e_{1}^{\alpha_{02}+\alpha_{03}+\alpha_{11}+\alpha_{14}} e_{2}^{\alpha_{01}+\alpha_{04}+\alpha_{12}+\alpha_{13}} e_{3}^{\alpha_{01}+\alpha_{04}+\alpha_{12}+\alpha_{13}} e_{4}^{\alpha_{02}+\alpha_{03}+\alpha_{11}+\alpha_{14}}\right) .
\end{aligned}
$$

To ensure that $s_{0} s_{1}$ does not have order 2 , it is desirable that one of $\alpha_{02}+\alpha_{03}+\alpha_{11}+$ $\alpha_{14}$ or $\alpha_{01}+\alpha_{04}+\alpha_{12}+\alpha_{13}$ be not equal to zero. The equalities derived earlier reduce these both to $\alpha_{01}-\alpha_{02}-\alpha_{11}+\alpha_{12} \neq 0$.

This has solutions over $\mathbf{Z}$. For example, we can choose $\alpha_{01}=1, \alpha_{02}=\alpha_{11}=$ $\alpha_{12}=0$, yielding $s_{0}=\sigma_{0} e_{1} e_{2}^{-1}$ and $s_{1}=\sigma_{1}$. Note then that $\left(s_{0} s_{1}\right)^{2}=e_{1}^{-1} e_{2} e_{3} e_{4}^{-1}$. This solution can be projected onto a solution to the corresponding equations over $\mathbf{Z}_{p}$. Then, $s_{0} s_{1}$ has order $2 p$, and $\left\langle s_{0}, s_{1}\right\rangle$ is the group of the $2 p$-gon.

In general, there will be far fewer linear equalities than variables $\alpha_{i, j}$. It seems likely that the construction will work often (in some sense)-unless the polytope $\mathcal{P}(\Gamma)$ has some special structure that ensures that the inequalities cannot be satisfied simultaneously with the equalities.

In support of this conjecture, the construction was attempted on all nondegenerate rank 3 polytopes in the Atlas of Small Regular Polytopes [6] with groups of order up to 336 . Of the 456 polyhedra considered, the construction could be applied to all but nine of them to yield infinite families (parametrised by $p$ ) of covering polytopes. The nine for which the construction failed are precisely those with a finite universal cover, namely, the platonic solids and their nondegenerate quotients.

Some scripts, written in the language of the GAP computer algebra system [13], are available from http://www.dr-mikes-maths.com/polyext.html. These scripts should be useful to anyone wishing to apply the construction to particular polytopes.

\section{A More General Scenario}

Not every extension of a polytope's automorphism group by a direct product of cyclic groups can be constructed using the construction considered so far. In general, if $\Gamma=\left\langle\sigma_{0}, \ldots, \sigma_{n-1}\right\rangle$ and $\Gamma \ltimes V=\left\langle s_{0}, \ldots, s_{n-1}\right\rangle$ are string C-groups, where $s_{i}=\sigma_{i} u_{i}$ for some elements $u_{i}$ of $U$, all we can say is that $\Gamma$ acts on the elements of $U$, not necessarily on its generators.

It is beyond the scope of this article to explore this more general situation in detail. However, one theorem and an interesting corollary will be proven. 
Theorem 5.1 Let $\Gamma=\left\langle\sigma_{0}, \ldots, \sigma_{n-1}\right\rangle$ and $W=\left\langle s_{0}, \ldots, s_{n-1}\right\rangle \cong \Gamma \ltimes V$ be string $C$-groups, where $V \cong Z_{p}^{k}$. Let $u_{i} \in V=\left\langle e_{1}, \ldots, e_{k}\right\rangle$ be such that $s_{i}=\sigma_{i} u_{i}$, and let $R$ be a set of words on the $\sigma_{i}$, a complete set of defining relations for the group $\Gamma$. Then

$$
V=\left\langle\left(\prod_{j=1}^{l} u_{i_{j}}^{\sigma_{i_{j+1}} \ldots \sigma_{i_{l}}}\right)^{g}: \sigma_{i_{1}} \ldots \sigma_{i_{l}} \in R, g \in \Gamma\right\rangle .
$$

Proof $V$ certainly contains the claimed generating set. Since $V$ is a subgroup of $\left\langle s_{0}, \ldots, s_{n-1}\right\rangle$, any element of $V$ must equal

$$
\prod_{j=1}^{t} s_{i_{j}}=\prod_{j=1}^{t} \sigma_{i_{j}} u_{i_{j}}=\prod_{j=1}^{t} \sigma_{i_{j}} \prod_{j=1}^{t} u_{i_{j}}^{\sigma_{i_{j+1}} \cdots \sigma_{i_{l}}}=\prod_{j=1}^{t} u_{i_{j}}^{\sigma_{i_{j+1}} \cdots \sigma_{i_{l}}}
$$

for some $i_{j}, j=1, \ldots, t$. If $w_{0}=\prod_{j=1}^{t} \sigma_{i j}=1$, then it can be reduced to the empty word $w_{q}$ by a sequence of insertions and deletions of elements of $R$, yielding a sequence of words $w_{0}, \ldots, w_{q}$, all equal to the identity in $\Gamma$. Let

$$
w_{p}=\prod_{j=1}^{t_{p}} \sigma_{i_{p, j}}
$$

and suppose first that $w_{p+1}$ is obtained from $w_{p}$ by insertion of $\prod_{j=1}^{k} \sigma_{i_{j}} \in R$. Then,

$$
w_{p+1}=\prod_{j=1}^{\alpha_{p}} \sigma_{i_{p, j}} \prod_{j=1}^{k} \sigma_{i_{j}} \prod_{j=\alpha_{p}+1}^{t_{p}} \sigma_{i_{p, j}}
$$

Then,

$$
\begin{aligned}
\prod_{j=1}^{t_{p}} s_{i_{p, j}} & =\prod_{j=1}^{\alpha_{p}} s_{i_{p, j}} \prod_{j=1}^{l} \sigma_{i_{j}} \prod_{\alpha_{p}+1}^{t_{p}} s_{i_{p, j}} \\
& =\prod_{j=1}^{\alpha_{p}} s_{i_{p, j}} \prod_{j=1}^{l} s_{i_{j}} u_{i_{j}}^{-1} \prod_{\alpha_{p}+1}^{t_{p}} s_{i_{p, j}} \\
& =\prod_{j=1}^{\alpha_{p}} s_{i_{p, j}} \prod_{j=1}^{l} s_{i_{j}} \prod_{j=1}^{l}\left(u_{i_{j}}^{\sigma_{i_{j+1}} \cdots \sigma_{i_{l}}}\right)^{-1} \prod_{\alpha_{p}+1}^{t_{p}} s_{i_{p, j}} \\
& =\prod_{j=1}^{\alpha_{p}} s_{i_{p, j}} \prod_{j=1}^{l} s_{i_{j}} \prod_{\alpha_{p}+1}^{t_{p}} s_{i_{p, j}}\left(\left(\prod_{j=1}^{l} u_{i_{j}}^{\sigma_{i j+1} \cdots \sigma_{i_{l}}}\right)^{g}\right)^{-1} \\
& =\prod_{j=1}^{t_{p+1}} s_{i_{p+1, j}}\left(\left(\prod_{j=1}^{l} u_{i_{j}}^{\sigma_{i_{j+1}} \cdots \sigma_{i_{l}}}\right)^{g}\right)^{-1},
\end{aligned}
$$


where $g$ is an element of $\Gamma$, since $V$ is Abelian. Similarly, if $w_{j+1}$ is obtained from $w_{j}$ by deletion of a word in $R$, then

$$
\prod_{j=1}^{t_{p}} s_{i_{p, j}}=\prod_{j=1}^{t_{p+1}} s_{i_{p+1, j}}\left(\prod_{j=1}^{l} u_{i_{j}}^{\sigma_{i_{j+1}} \ldots \sigma_{i_{l}}}\right)^{g}
$$

for some $g \in \Gamma, \sigma_{i_{1}} \cdots \sigma_{i_{l}} \in R$. Hence,

$$
\prod_{j=1}^{t} s_{i_{j}}=\prod_{j=1}^{t_{0}} s_{i_{0, j}}=\left(\prod_{j=1}^{t_{q}} s_{i_{q, j}}\right) v
$$

where

$$
v \in\left\langle\prod_{j=1}^{l} u_{i_{j}}^{\sigma_{i_{j+1}} \cdots \sigma_{i_{l}}}: \sigma_{i_{1}} \cdots \sigma_{i_{l}} \in R, g \in \Gamma\right\rangle .
$$

Since the left-hand side is an arbitrary element of $V$, and $w_{q}$ is the empty word, this proves the desired result.

This has the following interesting corollary.

Theorem 5.2 Let $\Gamma$ and $\Gamma \ltimes V$ be C-groups. Further, let $\Gamma$ be finite, and $V \cong \mathbf{Z}_{p}^{k}$. Finally, let $R$ be a complete set of defining relations for $\Gamma$. Then $|V| \leq p^{|R||\Gamma|}$. In particular, $V$ can not be arbitrarily large.

Proof From the previous theorem, $V$ is an Abelian group generated by at most $|R||\Gamma|$ elements, each of which has order at most $p$.

This does not imply that a polytope's automorphism group (a C-group) cannot be arbitrarily extended by direct products of cyclic groups, yielding larger and larger polytopes. For example, one could successively apply the construction with different characteristics $p$. The corollary also does not even imply that a C-group cannot be arbitrarily extended by direct products of cyclic groups with the same characteristic. For example, the $n$-gon is a 2 -fold quotient of a $2 n$-gon, which is in turn a 2 -fold quotient of a $4 n$-gon. However, the corollary implies that for some $k$, the group of the $2^{k} n$-gon will not be an extension of that of the $n$-gon by $\mathbf{Z}_{2}^{k}$.

The author would like to acknowledge the helpful comments Egon Schulte made on a draft of this article, as well as those of the anonymous referees.

\section{References}

1. Aschbacher, M.: Finite Group Theory. Cambridge University Press, Cambridge (2000)

2. Coxeter, H.S.M.: Ten toroids and fifty-seven hemi-dodecahedra. Geom. Dedic. 13, 87-99 (1982)

3. Coxeter, H.S.M.: A symmetrical arrangement of eleven hemi-icosahedra. Ann. Discrete Math. 20, 103-114 (1984) 
4. Grünbaum, B.: Regularity of graphs, complexes and designs. In: Problèmes Combinatoires et Théorie des Graphes. Coll. Int. CNRS, vol. 260, pp. 191-197 (1977)

5. Hartley, M.I.: Combinatorially regular Euler polytopes. Ph.D. Dissertation, University of Western Australia (1996)

6. Hartley, M.I.: An atlas of small regular abstract polytopes. Period. Math. Hung. 53, 149-156 (2006). Web-based atlas at http://www.abstract-polytopes.com/atlas/

7. Hartley, M.I., Leemans, D.: Quotients of a universal locally projective polytope of type $\{5,3,5\}$. Math. Z. 247, 663-674 (2004)

8. Hartley, M.I., Leemans, D.: On the thin regular geometries of rank four for the Janko group $J_{1}$. Innov. Incid. Geom. 1, 181-190 (2005)

9. Hartley, M.I., Leemans, D.: On locally spherical polytopes of type $\{5,3,5\}$. Discrete Math. 309, 247254 (2009)

10. Leemans, D., Vauthier, L.: An atlas of abstract regular polytopes for small groups. Aequ. Math. 72, 313-320 (2006)

11. McMullen, P., Schulte, E.: Abstract Regular Polytopes. Cambridge University Press, Cambridge (2002)

12. Monson, B., Schulte, E.: Modular reduction in abstract polytopes. Can. Math. Bull. 52, 425-450 (2009)

13. The GAP Group: GAP_Groups, Algorithms, and Programming, Version 4.4, http://www. gap-system.org (2005) 